\title{
Nonlinear State Estimation in Suspension Control Based on Takagi-Sugeno Model *
}

\author{
Nils Pletschen* Patrick Badur* \\ * Institute of Automatic Control (Prof. Dr.-Ing. B. Lohmann), \\ Technische Universität München, 85748 Garching, Germany \\ (e-mail: nils.pletschen@tum.de).
}

\begin{abstract}
We present a new nonlinear state estimation approach based on Kalman filter theory and Takagi-Sugeno (TS) modeling for an active vehicle suspension application in this paper. The nonlinear state equations of a so-called hybrid suspension configuration, which result from nonlinear spring and damping characteristics, are exactly represented by means of a continuoustime TS system, i. e. a convex combination of local linear state space models. We derive observer gain matrices for each linear subsystem on the basis of standard Kalman filter theory, before we construct the global observer for the overall nonlinear system. Convergence of the global observer is ensured in terms of linear matrix inequality conditions. We then study the estimation performance of the TS Kalman filter in simulations and experiments on a hybrid quarter-car test rig using a measured road profile as disturbance input. The approach achieves a high estimation accuracy of well above $90 \%$ in the simulation and $70-90 \%$ in the experiments.
\end{abstract}

Keywords: Active suspensions; Semi-active dampers; State estimation; Kalman filter;

Takagi-Sugeno model; Vehicle dynamics.

\section{INTRODUCTION}

Vehicle suspensions have been and still are subject to research and development in the automotive field. By integrating active and semi-active components into the suspension of production vehicles the primary conflict between ride comfort and ride safety due to an uneven road surface as well as the objective to meet constructional constraints can be eased, Hrovat (1997). In order to beneficially modify the vertical suspension dynamics by means of mechatronic elements, relevant information about the current driving state must be at least partially known. The information needed may range from solely the absolute body and the damper relative velocity, see e.g. Karnopp et al. (1974), up to the full vertical dynamic state vector if state feedback control is applied, see e. g. Hrovat (1997), Venhovens (1994). Since for practical and economical reasons measuring all of these quantities is not an option, estimation techniques have to be applied to calculate the system states from the available measurement signals.

Classical Kalman filter theory qualifies for application in linear suspension state estimation due to its optimality properties and its convenience in implementation, see Zarchan and Musoff (2005). Furthermore, as discussed in Venhovens (1994) the road height velocity of characteristic road disturbances may approximately be described by means of Gaussian white noise processes. Kalman filter design for active suspension control based on a linear plant model is discussed e. g. in Sharma et al. (1994) and Yu and Crolla (1998). However, when passive or semi-active dampers are part of the suspension configuration, their significant nonlinear force characteristics cannot be neglected without substantial deterioration in estimation quality.

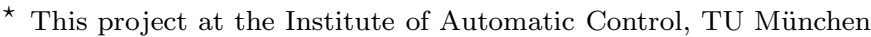
has been funded by the German Research Foundation (DFG).
}

Thus, a frequently employed strategy is to extend a linear suspension representation by a nonlinear damper model that generates a fictitious force input based on the estimated damper relative velocity, see e.g. Lindgärde (2002), Koch et al. (2010). General extensions of Kalman filter theory to nonlinear systems are the Extended (EKF) or the Unscented Kalman filter (UKF) variants, see e. g. Zarchan and Musoff (2005) and Simon (2006). Suspension control applications of these concepts may be found in Koch et al. (2010) and Fleps-Dezasse and Brembeck (2013). However, these approaches are computationally intensive.

In contrast, Takagi-Sugeno (TS) models (Takagi and Sugeno (1985)) aim at representing nonlinear dynamics by a smooth interpolation of linear models. Thereby, for a quite general class of nonlinear systems an exact description of the nonlinear dynamics can be obtained at least on a compact region of interest incorporating the origin by using the sector nonlinearity approach (Tanaka and Wang (2001)). Thus, we may apply linear control and observer theory upon the given linear models, to construct a global controller or observer for the overall nonlinear system. Stability analysis as well as control and observer design for TS systems may then be defined as linear matrix inequality (LMI) problems, see e. g. Tanaka and Wang (2001).

In this paper we combine exact TS modeling with the amenities of linear Kalman filter theory to allow for state feedback in suspension control. Therefore, we represent the given nonlinear model of a hybrid suspension system in a quarter-car framework as a family of local linear state space models. Then, we design local Kalman filters to estimate the states for each of these linear subsystems, before the global state estimator for the nonlinear vehicle suspension is derived. Simon (2003) describes a similar approach for fuzzy discrete time systems, where 
optimality properties and simulation results are discussed. However, in this work we study the continuous-time case of state estimation, since an exact transformation of the given nonlinear plant model into the discrete-time TS representation via the sector nonlinearity approach is not possible without inducing discretization errors. Moreover, the nonlinear functions involved in the modeling of the vehicle suspension studied here are rather given in terms of identified nonlinear force characteristics than by using analytic relationships, cf. Georg et al. (2012).

We organized the paper as follows: Section 2 gives a general introduction to TS modeling and observer design. In Section 3 the TS representation of the hybrid suspension system is derived, before the TS Kalman filter for suspension state estimation is designed in Section 4. In Section 5 we present simulation and experimental results to study the estimation performance of the proposed concept.

\section{TAKAGI-SUGENO OBSERVER DESIGN}

\subsection{TS System Representation}

Consider an input-affine nonlinear system of the form

$$
\begin{aligned}
& \dot{\mathbf{x}}(t)=\mathbf{A}(\mathbf{x}) \mathbf{x}(t)+\mathbf{B}(\mathbf{x}) \mathbf{u}(t) \\
& \mathbf{y}(t)=\mathbf{C}(\mathbf{x}) \mathbf{x}(t)+\mathbf{D}(\mathbf{x}) \mathbf{u}(t) .
\end{aligned}
$$

In order to design a TS observer, system (1) is transformed into the Takagi-Sugeno model representation

$$
\begin{aligned}
\dot{\mathbf{x}}(t) & =\sum_{i=1}^{l} h_{i}(\boldsymbol{\theta}(t))\left[\mathbf{A}_{i} \mathbf{x}(t)+\mathbf{B}_{i} \mathbf{u}(t)\right] \\
\mathbf{y}(t) & =\sum_{i=1}^{l} h_{i}(\boldsymbol{\theta}(t))\left[\mathbf{C}_{i} \mathbf{x}(t)+\mathbf{D}_{i} \mathbf{u}(t)\right],
\end{aligned}
$$

where $h_{i}(\boldsymbol{\theta}(t))$ denotes the weighting function determining the share by which the $i$ th linear subsystem contributes to the overall system dynamics.

Therefore, each nonlinear relationship in (1) is handled separately. If the $n_{l}$ nonlinearities $f_{k}(\boldsymbol{\theta}(t))$ with $k \epsilon$ $\left\{1, \ldots, n_{l}\right\}$ lie within sectors resulting from linear subsystems, the sector nonlinearity approach allows for an exact representation of the original system. Therein, $\boldsymbol{\theta}(t)$ denotes the vector of premise variables, i. e. the quantities the nonlinear functions depend on. With the transformation

$$
f_{k}(\boldsymbol{\theta}(t))=\underline{f}_{k} \underbrace{\frac{\bar{f}_{k}-f_{k}(\boldsymbol{\theta}(t))}{\bar{f}_{k}-\underline{f}_{k}}}_{\mu_{k, 1}(\boldsymbol{\theta})}+\bar{f}_{k} \underbrace{\frac{f_{k}(\boldsymbol{\theta}(t))-\underline{f}_{k}}{\bar{f}_{k}-\underline{f}_{k}}}_{\mu_{k, 2}(\boldsymbol{\theta})}
$$

of the $k$ th nonlinearity $f_{k}(\boldsymbol{\theta}(t))$, the TS system can be formulated as a weighted convex sum of the linear subsystems given by the sector bounds. Therein, $\mu_{k, 1}(\boldsymbol{\theta}(t))$ and $\mu_{k, 2}(\boldsymbol{\theta}(t))$ denote the sector membership functions and $\underline{f}_{k}=\min \left(f_{k}(\boldsymbol{\theta}(t))\right)$ and $\bar{f}_{k}=\max \left(f_{k}(\boldsymbol{\theta}(t))\right)$ indicate the minimum and maximum sector bounds of the $k$ th nonlinearity, respectively. Hence, $\mu_{k, 1}(\boldsymbol{\theta}(t))$ and $\mu_{k, 2}(\boldsymbol{\theta}(t))$ give the fulfillment grade of $f_{k}(\boldsymbol{\theta}(t))$ with respect to $\underline{f}_{k}$ and $\bar{f}_{k}$, respectively. Since the sector bounds fully enclose the nonlinearity on the universe of discourse, $\sum_{j_{k}=1}^{2} \mu_{k, j_{k}}(\boldsymbol{\theta}(t))=1$ and $0 \leq \mu_{k, j_{k}}(\boldsymbol{\theta}(t)) \leq 1$ hold for the sector functions.

The $i$ th linear subsystem $\left(\mathbf{A}_{i}, \mathbf{B}_{i}, \mathbf{C}_{i}, \mathbf{D}_{i}\right)$ in $(2)$ is constructed by employing the corresponding combinations of minimum and maximum sector bounds instead of using the nonlinearities themselves and thus represents the nonlinear system at one 'extreme' configuration. Since a set of two sector membership functions is needed to describe each of the $n_{l}$ nonlinearities, for the number of linear subsystems $l=2^{n_{l}}$ holds. The degree of activation of each subsystem is then given by the aggregated weighting functions, e. g.

$$
h_{i}(\boldsymbol{\theta}(t))=\mu_{1, j_{1}}(\boldsymbol{\theta}(t)) \cdot \ldots \cdot \mu_{n_{l}, j_{n_{l}}}(\boldsymbol{\theta}(t))
$$

determined from one possible combination $i$ of the $n_{l}$ sector functions $\mu_{k, j_{k}}(\boldsymbol{\theta}(t))$ with $j_{k} \in\{1,2\}$. Thus, also the weighting functions satisfy the convex sum property

$$
\sum_{i=1}^{l} h_{i}(\boldsymbol{\theta}(t))=1 \quad \text { and } \quad 0 \leq h_{i}(\boldsymbol{\theta}(t)) \leq 1, \quad \forall i,
$$

a fact that facilitates stability analysis later on. In general, the premise variable $\boldsymbol{\theta}(t)$ may be composed of system states, inputs, and measurements. However, here we assume that $\boldsymbol{\theta}(t)$ consists of measured quantities and known inputs only and does not depend on estimated states.

The rationale above can easily be extended to a broad class of nonlinear functions. In particular, as shown in Bergsten (2001) this is true for nonlinear systems of the form

$$
\begin{aligned}
& \dot{\mathbf{x}}(t)=\mathbf{A}(\mathbf{x}, \mathbf{u}) \mathbf{x}(t)+\mathbf{B}(\mathbf{x}, \mathbf{u}) \mathbf{u}(t) \\
& \mathbf{y}(t)=\mathbf{C}(\mathbf{x}, \mathbf{u}) \mathbf{x}(t)+\mathbf{D}(\mathbf{x}, \mathbf{u}) \mathbf{u}(t)
\end{aligned}
$$

\subsection{Deterministic TS Observer Design}

Consider a time-invariant full-order observer for (2) of the form, see e.g. Bergsten (2001),

$$
\begin{aligned}
& \dot{\hat{\mathbf{x}}}(t)=\sum_{i=1}^{l} h_{i}(\boldsymbol{\theta}(t))\left[\mathbf{A}_{i} \hat{\mathbf{x}}(t)+\mathbf{B}_{i} \mathbf{u}(t)+\mathbf{L}_{i}(\mathbf{y}(t)-\hat{\mathbf{y}}(t))\right] \\
& \hat{\mathbf{y}}(t)=\sum_{i=1}^{l} h_{i}(\boldsymbol{\theta}(t))\left[\mathbf{C}_{i} \hat{\mathbf{x}}(t)+\mathbf{D}_{i} \mathbf{u}(t)\right] .
\end{aligned}
$$

Therein, $\mathbf{L}_{i}$ denotes the observer gain matrix of the $i$ th linear subsystem and $h_{i}(\boldsymbol{\theta}(t))$ coincides with the $i$ th aggregated membership function of the system model (2). The objective of the observer (7) is to estimate the states of the system (2). This is the case, if the error dynamics $\dot{\mathbf{e}}=\dot{\mathbf{x}}-\dot{\hat{\mathbf{x}}}$ are asymptotically stable. Following Tanaka and Wang (2001), the error dynamics yield

$$
\dot{\mathbf{e}}=\sum_{i=1}^{l} \sum_{j=1}^{l} h_{i}(\boldsymbol{\theta}(t)) h_{j}(\boldsymbol{\theta}(t))\left[\mathbf{A}_{i}-\mathbf{L}_{i} \mathbf{C}_{j}\right] \mathbf{e} .
$$

Lyapunov theory then shows that the error dynamics are asymptotically stable, if there exists a common positive definite matrix $\widetilde{\mathbf{P}}=\widetilde{\mathbf{P}}^{T}>0$, such that

$$
\begin{aligned}
\mathbf{H}_{i i}^{T} \widetilde{\mathbf{P}}+\widetilde{\mathbf{P}} \mathbf{H}_{i i} & <0, \\
\left(\mathbf{H}_{i j}+\mathbf{H}_{j i}\right)^{T} \widetilde{\mathbf{P}}+\widetilde{\mathbf{P}}\left(\mathbf{H}_{i j}+\mathbf{H}_{j i}\right) & <0, \quad i<j,
\end{aligned}
$$

where $\mathbf{H}_{i j}=\left(\mathbf{A}_{i}-\mathbf{L}_{i} \mathbf{C}_{j}\right)$ and $i, j \in\{1, \ldots, l\}$. The constraints given by the LMIs in (9) thus imply that $\mathbf{L}_{i}$ not only stabilizes $\left(\mathbf{A}_{i}^{T}, \mathbf{C}_{i}^{T}\right)$, but also $\left(\mathbf{A}_{i}^{T}, \mathbf{C}_{j}^{T}\right)$.

The observer gains $\mathbf{L}_{i}$ can be either determined separately for each linear subsystem by applying linear estimation theory or may be designed globally, see e.g. Tanaka and Wang (2001). While the LMI constraints in (9) in the former case subsequently check whether the error dynamics (8) are stable or not, in the latter case the $\mathbf{L}_{i}$ are part of the LMI solution designed such that convergence is ensured and for example a desired decay rate is obtained. 


\subsection{Stochastic TS Observer Design}

However, physical dynamic systems are not only influenced by means of known control inputs $\mathbf{u}(t)$, but may also be subject to stochastic disturbances. Thus, the extension of the TS state space model (2) according to the linear state equation representation as given in Maybeck (1979) by adding noise processes yields

$$
\begin{aligned}
& \dot{\mathbf{x}}(t)=\sum_{i=1}^{l} h_{i}(\boldsymbol{\theta}(t))\left[\mathbf{A}_{i} \mathbf{x}(t)+\mathbf{B}_{i} \mathbf{u}(t)+\mathbf{G}_{i} \mathbf{w}(t)\right] \\
& \mathbf{y}(t)=\sum_{i=1}^{l} h_{i}(\boldsymbol{\theta}(t))\left[\mathbf{C}_{i} \mathbf{x}(t)+\mathbf{D}_{i} \mathbf{u}(t)+\mathbf{v}(t)\right] .
\end{aligned}
$$

Therein, $\mathbf{w}(t)$ and $\mathbf{v}(t)$ denote the zero-mean Gaussian white process and measurement noise, respectively, characterized by their covariance kernels

$$
\begin{aligned}
E\left\{\mathbf{w}(t) \mathbf{w}^{T}(\tau)\right\} & =\mathbf{Q} \delta(t-\tau), & & \mathbf{Q}=\mathbf{Q}^{T}>0, \\
E\left\{\mathbf{v}(t) \mathbf{v}^{T}(\tau)\right\} & =\mathbf{R} \delta(t-\tau), & & \mathbf{R}=\mathbf{R}^{T}>0,
\end{aligned}
$$

where $E\{\cdot\}$ and $\delta(t-\tau)$ represent the expectation value operator and the Dirac delta, respectively. Furthermore, $\mathbf{w}(t)$ and $\mathbf{v}(t)$ are assumed to be uncorrelated.

Since the Kalman filter is of the same structure as (7) and due to its optimality properties in case of stochastically disturbed linear systems, we aim at determining $\mathbf{L}_{i}$ based on Kalman filter theory. Originally, the Kalman-Bucy filter, see Kalman and Bucy (1961), continuously calculates the expected value $\hat{\mathbf{x}}(t)$ of the true system state $\mathbf{x}(t)$ and its error covariance $\mathbf{P}(t)$. This results in a time-variant gain matrix $\mathbf{L}(t)$, which converges to a constant steadystate gain matrix $\mathbf{L}=$ const. if the linear system model is time-invariant. For the ease of implementation and in order to keep a time-invariant observer gain approach, the observer gain $\mathbf{L}_{i}$ in (7) for each linear subsystem $i$ is determined by the steady-state solution of the time-variant Kalman filter, see e.g. Simon (2006). Assuming the pairs $\left(\mathbf{A}_{i}, \mathbf{C}_{i}\right)$ to be detectable and $\left(\mathbf{A}_{i}, \mathbf{G}_{i}\right)$ to be stabilizable with $i \in\{1, \ldots, l\}$, for each linear subsystem $i$ one may find the steady-state Kalman observer gain matrix

$$
\mathbf{L}_{i}=\mathbf{P}_{i} \mathbf{C}_{i}^{T} \mathbf{R}^{-1},
$$

where $\mathbf{P}_{i}$ is the symmetric and positive definite solution of the algebraic matrix Riccati equation

$$
\mathbf{0}=\mathbf{A}_{i} \mathbf{P}_{i}+\mathbf{P}_{i} \mathbf{A}_{i}^{T}+\mathbf{G}_{i} \mathbf{Q} \mathbf{G}_{i}^{T}-\mathbf{P}_{i} \mathbf{C}_{i}^{T} \mathbf{R}^{-1} \mathbf{C}_{i} \mathbf{P}_{i} .
$$

In the following, a TS Kalman filter is utilized for state estimation in an actively controlled suspension application.

\section{VEHICLE SUSPENSION MODEL}

In this work, we address the so-called hybrid suspension configuration that has been studied e.g. in Koch et al. (2011) and Pletschen et al. (2013). This set-up consists of a slow-active spring mount adjustment, i. e. an actuator with a bandwidth of up to $5 \mathrm{~Hz}$ in series to the primary spring, in combination with a continuously variable damper, see Fig. 1 (right). The hybrid suspension strut is able to unite the respective advantages of active (high performance potential) and semi-active (low power demand) suspension components. For the analysis of the vertical motion of vehicle body and wheel mass quarter-car models can be used to study the major dynamic effects in the frequency range below $25 \mathrm{~Hz}$, Mitschke and Wallentowitz (2004).
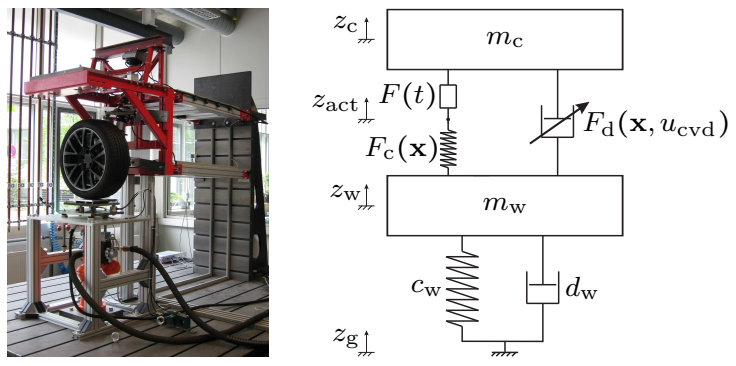

Fig. 1. Test rig (le.) and model of hybrid quarter-car (ri.)

Due to several nonlinear force elements, the quarter-car equations of motion can be written as a nonlinear state space model of the form (6). In the following, the nonlinear model of the quarter-car test rig is represented in TS model description, where the predominant nonlinear effects are taken into account. Then, we calculate the TS Kalman gain matrices for each linear subsystem from (13), before we determine the global observer and verify its stability.

\subsection{Quarter-Car Test Rig with Hybrid Suspension Strut}

On the basis of the suspension components, the kinematics, the actuators and the sensor architecture of a current upperclass sedan, a quarter-car test rig has been designed for experimental analysis of suspension control concepts, see Fig. 1 (left). For accurate simulation and control input calculation, the parameters and major nonlinear effects have been identified at the test rig and are taken into account, see Koch et al. (2011). Nonlinearities within the force transfer between chassis and wheel mass result from

- a progressive spring characteristic due to the included compression and tension stops,

- a semi-active damper with degressive force-velocity characteristics as well as an asymmetric behavior in the compression and the tension direction,

- Coulomb friction, and

- an inclined position of the suspension strut, and thus a kinematic transmission factor (Matschinsky (1998))

$$
i_{\mathrm{dyn}}=\frac{\left(\dot{z}_{\mathrm{c}}-\dot{z}_{\mathrm{w}}\right)^{(\text {strut })}}{\left(\dot{z}_{\mathrm{c}}-\dot{z}_{\mathrm{w}}\right)}=i_{0}-i_{1}\left(z_{\mathrm{c}}-z_{\mathrm{w}}\right)
$$

between the suspension strut plane and wheel plane that changes linearly with the suspension deflection.

The nonlinear behavior of the tire may be described by a linear-quadratic stiffness including possible wheel lift-off and a frequency-dependent tire damping characteristic.

The passive suspension configuration is realized by keeping the spring mount adjustment at a constant level and applying a constant current $I_{\mathrm{d}, \mathrm{p}}$ to the damper valves such that a medium damper characteristic results. Hence,

$$
f_{\mathrm{c}, \mathrm{p}}=\frac{1}{2 \pi} \sqrt{\frac{c_{\mathrm{c}}}{m_{\mathrm{c}}}} \approx 1.1 \mathrm{~Hz} \quad \text { and } \quad D_{\mathrm{c}, \mathrm{p}}=\frac{d_{\mathrm{c}}}{2 \sqrt{c_{\mathrm{c}} m_{\mathrm{c}}}} \approx 0.21
$$

are attained as undamped natural frequency and damping ratio of the body mass, respectively.

\subsection{Suspension Model in TS System Representation}

Based on the quarter-car model of the hybrid suspension configuration in Fig. 1 (right), the equations of motion for the chassis mass $m_{\mathrm{c}}$ and the wheel mass $m_{\mathrm{w}}$ are derived by applying Newton's law. Together with the state vector 
$\mathrm{x}(t)=\left[z_{\mathrm{c}}-z_{\mathrm{w}}, \dot{z}_{\mathrm{c}}, z_{\mathrm{w}}-z_{\mathrm{g}}, \dot{z}_{\mathrm{w}}\right]^{T}$ and the input vector $\mathbf{u}(t)=\left[u_{\mathrm{hy}}, u_{\mathrm{cvd}}, u_{\mathrm{d}}\right]^{T}$ these can be transformed into a fourth-order nonlinear state space model. While the deflection of the hydraulic actuator $u_{\text {hy }}(t)$, which manipulates the spring mount according to $z_{\mathrm{act}}(t)=z_{\mathrm{c}}(t)-u_{\mathrm{hy}}(t) / i_{\mathrm{dyn}}(t)$, and the valve current of the semi-active damper $u_{\mathrm{cvd}}(t)=$ $I_{\mathrm{d}}(t)$ serve as control inputs, $u_{\mathrm{d}}(t)=\dot{z}_{\mathrm{g}}(t)$ symbolizes the road-induced disturbance.

Furthermore, the output vector $\mathbf{y}(t)=\left[\ddot{z}_{\mathrm{c}}, \ddot{z}_{\mathrm{w}}, z_{\mathrm{cw}}\right]^{T}$ contains the chassis and the wheel acceleration $\ddot{z}_{\mathrm{c}}$ and $\ddot{z}_{\mathrm{w}}$ as well as the suspension travel $z_{\mathrm{cw}}=z_{\mathrm{c}}-z_{\mathrm{w}}$, and hence all of the measurement signals that are frequently available in production vehicles equipped with mechatronic suspension systems. However, the damper relative velocity $\dot{z}_{\mathrm{cw}}=\dot{z}_{\mathrm{c}}-$ $\dot{z}_{\mathrm{w}}$ is usually needed for the control of the semi-active damper. Thus, it is generated from the available measurement signals by applying a filter-based velocity estimation approach described in detail in Koch et al. (2010). The resulting velocity estimate has no phase delay and is of high estimation accuracy, such that the extended measurement vector $\tilde{\mathbf{y}}(t)=\left[\ddot{z}_{\mathrm{c}}, \ddot{z}_{\mathrm{w}}, z_{\mathrm{cw}}, \dot{z}_{\mathrm{cw}}\right]^{T}$ is obtained.

The nonlinear spring and damping force characteristics are represented by $F_{\mathrm{c}}(\mathbf{x})$ and $F_{\mathrm{d}}\left(\mathbf{x}, u_{\mathrm{cvd}}\right)$, respectively, where the nonlinear effect of the kinematic transmission ratio $i_{\text {dyn }}$ is already taken into account. It is important to note that $F_{\mathrm{c}}(\mathbf{x})$ and $F_{\mathrm{d}}\left(\mathbf{x}, u_{\mathrm{cvd}}\right)$ are rather given in terms of measured spring and damper characteristic maps than in terms of analytical relationships. Also, to incorporate Coulomb friction effects existent within the damper strut in $F_{\mathrm{d}}\left(\mathrm{x}, u_{\mathrm{cvd}}\right)$, we employ a friction model of the form

$$
F_{\mathrm{f}}(\mathbf{x})=\widetilde{F}_{\mathrm{f}} \cdot \tanh \left(k_{\mathrm{f}} \cdot v_{\mathrm{f}}\right)
$$

to realize smooth zero crossings. Thereby, $\widetilde{F}_{\mathrm{f}}$ is the size of the friction force, $k_{\mathrm{f}}$ is a scaling factor and $v_{\mathrm{f}}=\dot{z}_{\mathrm{cw}}$ holds.

While the progressive spring force $F_{\mathrm{c}}(\mathbf{x})$ does only depend on the state vector, in the semi-active case the degressive damper force $F_{\mathrm{d}}\left(\mathbf{x}, u_{\mathrm{cvd}}\right)$ is also influenced by the damper valve current input. Fig. 2 shows the nonlinear force characteristics. The effect of the kinematic transmission ratio $i_{\mathrm{dyn}}(t)$ is already incorporated for the spring force, while for the normalized damping force ${ }^{1}$ only the constant ratio $i_{0}$ is applied. The black solid lines represent the nonlinear force characteristics of the spring and the passive damper, while the black dashed lines in the damper force map show the semi-active characteristics for several constant settings of the damper valve current. The sectors $f_{1}(\boldsymbol{\theta}(t)) \in\left[\underline{f}_{1}, \bar{f}_{1}\right]$ and $f_{2}(\boldsymbol{\theta}(t)) \in\left[\underline{f}_{2}, \bar{f}_{2}\right]$, which are to be

1 For confidentiality reasons the damper force characteristics are given in terms of the normalized damper force map $F_{\mathrm{d}, \text { norm }}\left(\mathbf{x}, u_{\mathrm{cvd}}\right)$.
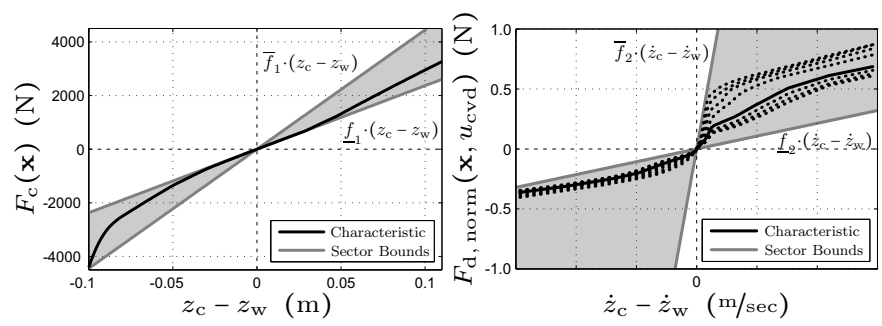

Fig. 2. Nonlinear spring force (left) and normalized damping force map (right) with their respective sectors determined in the following, are already illustrated here in Fig. 2 with respect to the force level by the gray lines.

To obtain the TS model representation of the hybrid suspension system, the nonlinear spring and damping forces are equally represented by

$$
\begin{aligned}
F_{\mathrm{c}}(\mathbf{x}) & =\tilde{c}_{\mathrm{c}}(\mathbf{x}) \cdot\left(z_{\mathrm{c}}-z_{\mathrm{w}}\right), \\
F_{\mathrm{d}}\left(\mathbf{x}, u_{\mathrm{cvd}}\right) & =\tilde{d}_{\mathrm{c}}\left(\mathbf{x}, u_{\mathrm{cvd}}\right) \cdot\left(\dot{z}_{\mathrm{c}}-\dot{z}_{\mathrm{w}}\right),
\end{aligned}
$$

where $\tilde{c}_{\mathrm{c}}(\mathbf{x})$ and $\tilde{d}_{\mathrm{c}}\left(\mathbf{x}, u_{\mathrm{cvd}}\right)$ are state- and input-dependent overall stiffness and damping coefficients, respectively. Hence, the nonlinear state space model can be reformulated, which together with the output equation results in (19) on the following page. Since tire damping usually is very small, i. e. $d_{\mathrm{w}}=0$, solely a linear stiffness $c_{\mathrm{w}}$ is assumed to describe the tire characteristics in the TS model.

We choose the nonlinearity $f_{1}(\boldsymbol{\theta}(t))$ to be the nonlinear stiffness coefficient $\tilde{c}_{\mathrm{c}}(\mathbf{x})$, which is only a function of the suspension deflection $z_{\mathrm{cw}}$. The second nonlinearity $f_{2}(\boldsymbol{\theta}(t))$ then corresponds to the nonlinear damping coefficient $\tilde{d}_{\mathrm{c}}\left(\mathbf{x}, u_{\mathrm{cvd}}\right)$, which depends on the damper relative velocity $\dot{z}_{\mathrm{cw}}$, the damper valve current $u_{\mathrm{cvd}}$, and on the suspension deflection $z_{\mathrm{cw}}$ due to the dynamic transmission ratio in (15). Thus, $\boldsymbol{\theta}(t)=\left[z_{\mathrm{cw}}, \dot{z}_{\mathrm{cw}}, u_{\mathrm{cvd}}\right]^{T}$ holds. $f_{1}(\boldsymbol{\theta}(t))$ and $f_{2}(\boldsymbol{\theta}(t))$ are then determined by rearranging (17) and (18), where we treat the singularities occuring in $z_{\mathrm{cw}}, \dot{z}_{\mathrm{cw}}=0$ by averaging the both-sided limit value

$$
\begin{aligned}
& \tilde{c}_{\mathrm{c}}\left(z_{\mathrm{cw}}=0\right)=\frac{1}{2}\left(\lim _{z_{\mathrm{cw}} \rightarrow 0^{-}} \frac{F_{\mathrm{c}}(\mathbf{x})}{z_{\mathrm{c}}-z_{\mathrm{w}}}+\lim _{z_{\mathrm{cw}} \rightarrow 0^{+}} \frac{F_{\mathrm{c}}(\mathbf{x})}{z_{\mathrm{c}}-z_{\mathrm{w}}}\right), \\
& \tilde{d}_{\mathrm{c}}\left(\dot{z}_{\mathrm{cw}}=0\right)=\frac{1}{2}\left(\lim _{\dot{z}_{\mathrm{cw}} \rightarrow 0^{-}} \frac{F_{\mathrm{d}}\left(\mathbf{x}, u_{\mathrm{cvd}}\right)}{\dot{z}_{\mathrm{c}}-\dot{z}_{\mathrm{w}}}+\lim _{\dot{z}_{\mathrm{cw}} \rightarrow 0^{+}} \frac{F_{\mathrm{d}}\left(\mathbf{x}, u_{\mathrm{cvd}}\right)}{\dot{z}_{\mathrm{c}}-\dot{z}_{\mathrm{w}}}\right) .
\end{aligned}
$$

The resulting nonlinear stiffness and damping coefficient maps are depicted in Fig. 3. Thereby, the passive damping coefficient is given in terms of the black solid line and the damping coefficients of the softest and the hardest damper characteristic are illustrated by black dashed lines. Applying the suspension model parameters given in Table 1 to (2), the TS representation of the suspension model can be constructed. Due to $n_{l}=2$ nonlinear terms the TS model consists of $l=2^{2}=4$ linear subsystems. Thereby, each local linear submodel $i$ results from one combination of the sector bounds as specified in Table 2 .

\section{DESIGN OF SUSPENSION OBSERVER}

The aim of the observer is to estimate the states of the full-scale suspension model which includes all the nonlinearities identified at the quarter-car test rig, see Section 3.1. This is done by means of a full-order TS observer based on the TS representation of the suspension model derived in Section 3.2 using Kalman filter theory.
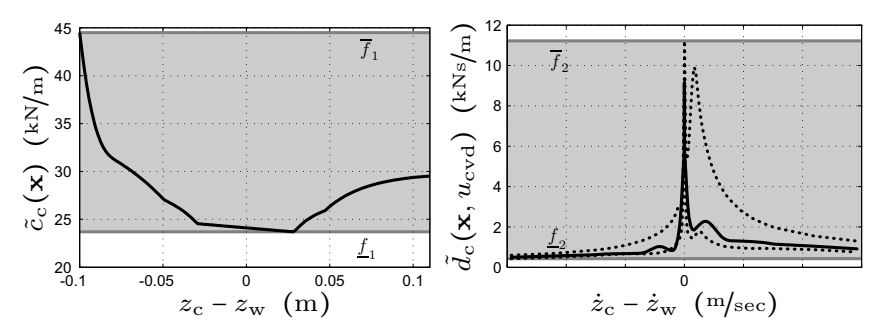

Fig. 3. Nonlinear stiffness (left) and damping coefficient map (right) with their sector bounds 


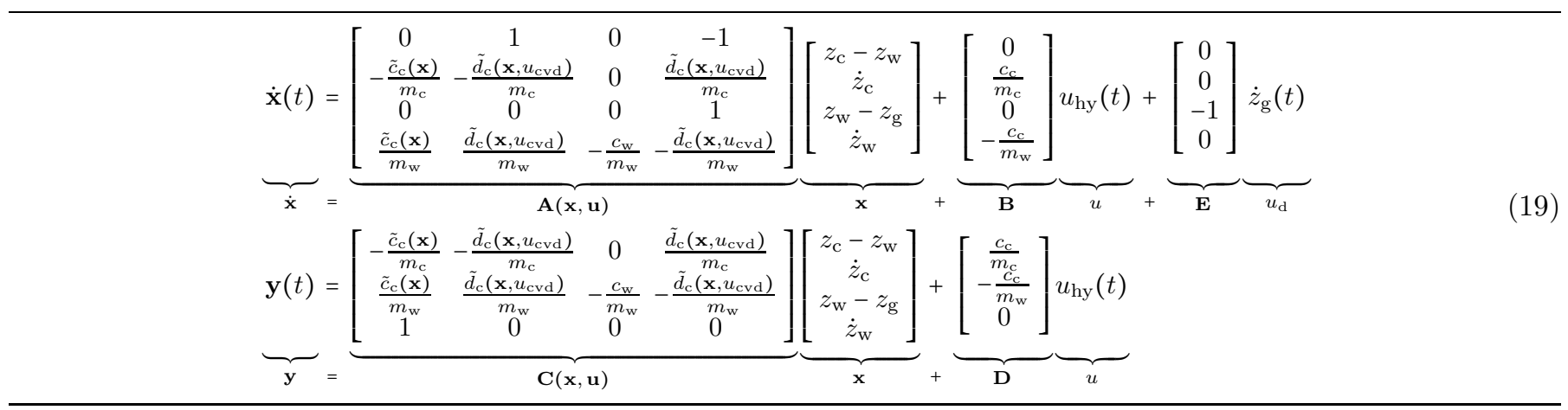

Table 1. TS suspension model parameters

\begin{tabular}{cccc}
\hline Parameter & Symbol & Value & Unit \\
\hline Sprung mass & $m_{\mathrm{c}}$ & $\approx 500$ & $\mathrm{~kg}$ \\
Unsprung mass & $m_{\mathrm{w}}$ & $\approx 70$ & $\mathrm{~kg}$ \\
Sector bounds (stiffness) & $\tilde{c}_{\mathrm{c}} \in\left[\underline{f}_{1}, \bar{f}_{1}\right]$ & $\approx[23,45]$ & $\mathrm{kN} / \mathrm{m}$ \\
Sector bounds (damping) & $\tilde{d}_{\mathrm{c}} \in\left[\underline{f}_{2}, \bar{f}_{2}\right]$ & $\approx[0.4,12]$ & $\mathrm{kNs} / \mathrm{m}$ \\
Stiffness effective for $u_{\mathrm{hy}}$ & $c_{\mathrm{c}}$ & $\approx 32$ & $\mathrm{kN} / \mathrm{m}$ \\
Tire stiffness & $c_{\mathrm{w}}$ & $\approx 380$ & $\mathrm{kN} / \mathrm{m}$ \\
Tire damping & $d_{\mathrm{w}}$ & $=0$ & $\mathrm{Ns} / \mathrm{m}$ \\
\hline
\end{tabular}

\subsection{Takagi-Sugeno Steady-State Kalman Filter}

For the design of the TS Kalman filter the representation

$$
\begin{aligned}
& \dot{\mathbf{x}}(t)=\sum_{i=1}^{l=4} h_{i}(\boldsymbol{\theta}(t))\left[\mathbf{A}_{i} \mathbf{x}(t)+\mathbf{B} u_{\mathrm{hy}}(t)+\mathbf{G w}(t)\right] \\
& \mathbf{y}(t)=\sum_{i=1}^{l=4} h_{i}(\boldsymbol{\theta}(t))\left[\mathbf{C}_{i} \mathbf{x}(t)+\mathbf{D} u_{\mathrm{hy}}(t)+\mathbf{v}(t)\right]
\end{aligned}
$$

of (19) is considered, where $\mathbf{A}_{i} \in \mathbb{R}^{4 \times 4}$ and $\mathbf{C}_{i} \in \mathbb{R}^{3 \times 4}$ are different for each linear subsystem $i \in\{1, \ldots, 4\}$, while $\mathbf{B} \in \mathbb{R}^{4 \times 1}$ and $\mathbf{D} \in \mathbb{R}^{3 \times 1}$ are the same for each of them. Model uncertainties and the unknown road disturbance $u_{\mathrm{d}}(t)=$ $\dot{z}_{\mathrm{g}}(t)$ are treated as process noise $\mathbf{w}(t)$. Furthermore, $\mathbf{G}=\mathbf{I}$ is chosen, where $\mathbf{I}$ indicates the identity matrix of appropriate dimension, corresponding to an identical intensity of the process noise in each linear subsystem. As all the pairs $\left(\mathbf{A}_{i}, \mathbf{C}_{i}\right)$ have been identified observable and $\left(\mathbf{A}_{i}, \mathbf{G}\right)$ is controllable, the requirements of detectability and stabilizability posed in Section 2.3 are met.

Thus, for each linear subsystem a steady-state Kalman gain $\mathbf{L}_{i}$ can be calculated from (13). The solution of the algebraic Riccati equation and the Kalman gains are both controlled by means of the covariance kernels $\mathbf{Q}$ and $\mathbf{R}$ of the process and the measurement noise, respectively. These design parameters are tuned within the optimization procedure described subsequently in Section 4.2.

As $\boldsymbol{\theta}(t)=\left[z_{\mathrm{cw}}, \dot{z}_{\mathrm{cw}}, u_{\mathrm{cvd}}\right]^{T}$, all premise variables are available from $\tilde{\mathbf{y}}(t)$ or the controller and thus the scheduling vector is independent of signals estimated by the observer.

Table 2. TS system configuration

\begin{tabular}{ccccc}
\hline Subsystem $i$ & 1 & 2 & 3 & 4 \\
\hline$\tilde{c}_{\mathrm{c}}(\mathbf{x})$ & $\bar{f}_{1}$ & $\bar{f}_{1}$ & $\underline{f}_{1}$ & $\underline{f}_{1}$ \\
$\tilde{d}_{\mathrm{c}}\left(\mathbf{x}, u_{\mathrm{cvd}}\right)$ & $\bar{f}_{2}$ & $\underline{f}_{2}$ & $\bar{f}_{2}$ & $\underline{f}_{2}$ \\
\hline
\end{tabular}

\subsection{Observer Tuning}

The tuning of the observer structure is carried out by varying the covariance matrices $\mathbf{Q}$ and $\mathbf{R}$, where all offdiagonal elements are chosen to be zero. The covariance entries on the diagonal are determined by means of a multiobjective optimization algorithm. Hence, for a given signal $\xi\left(t_{n}\right)$ on an equally spaced time set $T=\left\{t_{0}, t_{1}, \ldots, t_{N}\right\}$ the normalized root mean square error (NRMSE)

$$
\Delta_{\xi}=\frac{\sqrt{\frac{1}{N} \sum_{n=1}^{N}\left(\xi\left(t_{n}\right)-\hat{\xi}\left(t_{n}\right)\right)^{2}}}{\sqrt{\frac{1}{N} \sum_{n=1}^{N} \xi^{2}\left(t_{n}\right)}}
$$

is used to quantify the signal's estimation accuracy. Therein, the estimated value of $\xi\left(t_{n}\right)$ is denoted by $\hat{\xi}\left(t_{n}\right)$. As all quantities which are relevant for control purposes can be computed from the system states, the optimization problem stated in terms of the vector-valued objective function $\mathbf{J}(\eta)$ can be written as

$$
\min _{\eta} \mathbf{J}(\eta)=\min _{\eta}\left[\Delta_{z_{\mathrm{c}}-z_{\mathrm{w}}} \Delta_{\dot{z}_{\mathrm{c}}} \Delta_{z_{\mathrm{w}}-z_{\mathrm{g}}} \Delta_{\dot{z}_{\mathrm{w}}}\right]^{T} .
$$

Thereby, $\eta=\left[\begin{array}{lllllll}q_{1} & q_{2} & q_{3} & q_{4} & r_{1} & r_{2} & r_{3}\end{array}\right]^{T}$ denotes the vector of optimization variables and consists of the diagonal elements of $\mathbf{Q}$ and $\mathbf{R}$, such that $\Delta_{\xi}=\Delta_{\xi}(\eta)$ as defined in (22). For the observer application we choose

$$
\eta_{\text {com }}=\arg \min _{\eta}\|\mathbf{J}(\eta)\|,
$$

i. e. the parameter set that minimizes the 2-norm of the objective function $\mathbf{J}(\eta)$ and thus represents the best compromise of all Pareto-optimal parameter sets.

To avoid overestimation of the observer performance we use a generic road profile $z_{\mathrm{g}}(t)$ for the parameter optimization. As the road height velocity $\dot{z}_{\mathrm{g}}$ is the disturbance input to the model, white noise is used as excitation. To induce sufficiently large excitation the road parameters are chosen such that a highway road of medium quality results being traveled at a vehicle speed of $v_{\mathrm{veh}}=180 \mathrm{~km} / \mathrm{h}$. Simultaneously, this corresponds to a bad country road traveled at a lower velocity.

The observer tuning is conducted by means of the nonlinear full-scale model of the passive suspension, where the measured signals in the simulation are artificially exposed to measurement noise, whose intensity has been derived from sensor signals recorded at the test rig. Moreover, to only quantify estimation errors resulting from dynamic deviations, the initial observer state $\hat{\mathbf{x}}_{0}$ is chosen according to the initial state of the plant model $\mathbf{x}_{0}$ starting from its rest position, i. e. $\hat{\mathbf{x}}_{0}=\hat{\mathbf{x}}\left(t_{0}\right)=\mathbf{0}$ holds.

The optimization problem stated above is then solved by using the multi-objective genetic algorithm NSGA-II 
proposed in Deb et al. (2000). As shown in Table 3, the decline of the estimation accuracy $\Gamma_{\xi \text {, com }}=1-\Delta_{\xi \text {, com }}$ resulting from the best compromise of all parameter sets $\eta_{\text {com }}$ compared to the maximum achievable estimation quality $\Gamma_{\xi, \max }=1-\Delta_{\xi, \max }$ for each individual signal $\xi(t)$ is marginal. Not surprisingly the third state $z_{\mathrm{wg}}$ is estimated with lowest accuracy, since the unknown road disturbance $\dot{z}_{\text {g has }}$ an immediate impact and measurement information is neither available with regard to the road height nor to one of its derivatives.

Using Matlab's Robust Control Toolbox a matrix $\widetilde{\mathbf{P}}$ has been found for the parameter set $\eta_{\text {com }}$ satisfying the LMI constraints given in (9) based on the $\mathbf{L}_{i}$ according to (13), such that convergence of the global TS observer is ensured.

Table 3. Estimation performance on generic road profile: Maximum vs. best compromise

\begin{tabular}{ccccc}
\hline Quantity $\xi$ & $z_{\mathrm{c}}-z_{\mathrm{w}}$ & $\dot{z}_{\mathrm{c}}$ & $z_{\mathrm{w}}-z_{\mathrm{g}}$ & $\dot{z}_{\mathrm{w}}$ \\
\hline$\Gamma_{\xi, \max }$ & 0.980 & 0.905 & 0.783 & 0.961 \\
$\Gamma_{\xi, \text { com }}$ & 0.973 & 0.901 & 0.778 & 0.936 \\
\hline
\end{tabular}

\section{SIMULATION AND EXPERIMENTAL RESULTS}

For the estimation performance analysis of the TS Kalman filter in simulation and experiments, the nonlinear fullscale model of the hybrid quarter-car suspension and the quarter-car test rig as presented in Section 3 are used. A measurement of a real rough country road profile at a vehicle speed of $v_{\text {veh }}=50 \mathrm{~km} / \mathrm{h}$ serves as the road excitation. The evaluation is either done for the passive suspension configuration, i. e. $u_{\mathrm{cvd}}(t)=I_{\mathrm{d}, \mathrm{p}}=$ const. and $u_{\mathrm{hy}}(t)=$ $0 \mathrm{~m}=$ const., or for an actively controlled setting. In the controlled case, the reference-model based control concept presented in Koch et al. (2011) is employed. The approach solely relies on measurable quantities and directly provides separate control inputs $u_{\text {crd }}(t)$ and $u_{\text {hy }}(t)$.

In the simulation, the reference signals of the suspension states are immediately available from the full-scale model. As for the optimization, artificial measurement noise is added to the measurement signals fed to the TS observer. However, in the experiments reference signals for all state variables are not readily at hand. Thus, for reasons of analysis only, additional signals are recorded at the test rig. For instance, the absolute chassis position $z_{\mathrm{c}}$ is measured by means of a wire rope actuated position transducer mounted between the chassis mass and the inertial ground. Also, force sensors log the dynamic tire load $F_{\text {dyn }}$ between the tire and the ground and the vertical displacement $z_{\mathrm{g}}$ is recorded for actuator control purposes. The sampling time of the digital signal processing system is $T_{\mathrm{s}}=2 \mathrm{msec}$. To reduce measurement noise in the recorded signals, lowpass forward backward filtering with a cutoff frequency of $30 \mathrm{~Hz}$ is applied offline for the reference signals. While a reference for the first state, i. e. the suspension deflection, is directly available as a measurement signal, the reference signals of the chassis and wheel velocity are calculated by applying the filter-based velocity estimation approach already used for the damper relative velocity, see Section 3.2 , to the measurement signals of $z_{\mathrm{c}} / \ddot{z}_{\mathrm{c}}$ and $\left(z_{\mathrm{c}}-z_{\mathrm{cw}}\right) / \ddot{z}_{\mathrm{w}}$. The tire deflection reference $z_{\mathrm{wg}}$ is constructed from $z_{\mathrm{c}}, z_{\mathrm{g}}$ and $z_{\mathrm{cw}}$.

The simulation and measurement results for the passive suspension configuration are summarized in Table 4 and
Table 4. Estimation performance of passive configuration on rough country road profile at $v_{\mathrm{veh}}=50 \mathrm{~km} / \mathrm{h}$ : Simulation vs. Experiment

\begin{tabular}{ccccccc}
\hline & $\Gamma_{z_{\mathrm{c}}-z_{\mathrm{w}}}$ & $\Gamma_{\dot{z}_{\mathrm{c}}}$ & $\Gamma_{z_{\mathrm{w}}-z_{\mathrm{g}}}$ & $\Gamma_{\dot{z}_{\mathrm{w}}}$ & $\Gamma_{F_{\mathrm{dyn}}}$ & $\Gamma_{\dot{z}_{\mathrm{c}}-\dot{z}_{\mathrm{w}}}$ \\
\hline Simulation & 0.995 & 0.948 & 0.909 & 0.949 & 0.966 & 0.977 \\
Experiment & 0.932 & 0.801 & 0.660 & 0.804 & 0.701 & 0.874 \\
\hline
\end{tabular}

are illustrated by means of a spider chart in Fig. 4. In the simulation all relevant quantities are estimated with a NRMSE less than $10 \%$. Again, $z_{\mathrm{wg}}$ is the state with lowest estimation accuracy, since the unknown road disturbance has direct impact. Still, the performance is remarkable.

In case of the experimental results a slight degradation in estimation accuracy of about 5 to $15 \%$ for the quantities $z_{\mathrm{cw}}, \dot{z}_{\mathrm{c}}, \dot{z}_{\mathrm{w}}$ and $\dot{z}_{\mathrm{cw}}$ is noticeable. However, for the tirerelated quantities $z_{\mathrm{wg}}$ and $F_{\mathrm{dyn}}$ the performance decreases by about $25 \%$. This overall performance decline is due to the fact, that the actuator dynamics of the semi-active damper have not fully been included in the modeling for observer design. While the damper's valve current is available as a measurement and thus the dynamics of the electrical domain are inherently included, the force rise dynamics of the damper are not. If approximated by a first order lag element a mechanical time constant $T_{\text {mech }} \approx$ $10 \mathrm{msec}$ results to account for the hydraulic properties of the damper, see Koch et al. (2011).

Finally, the actively controlled suspension configuration is studied. A selected time interval of a measurement at the test rig is shown in the plots of Fig. 5. The upper five graphs depict the reference (black solid line), the estimate of the steady-state TS Kalman filter (blue dashed) and the respective estimation error (red solid) for the states and the dynamic tire load, respectively. In the lower plots of Fig. 5 the sector membership functions $\mu_{1, j_{1}}(\boldsymbol{\theta}(t))$ for the stiffness coefficient (solid lines) and $\mu_{2, j_{2}}(\boldsymbol{\theta}(t))$ for the damping coefficient (dashed lines), as well as the input signals $u_{\text {hy }}(t)$ and $u_{\text {cvd }}(t)$ (individually in compression and tension direction) are given. Note that half of the time period is depicted for the tire-related quantities to account for the high frequency dynamics included. The estimation performance for each individual signal lies within $5 \%$ of the $\Gamma$-values given in Table 4 .

\section{CONCLUSION}

The concept of Takagi-Sugeno observer design has been adopted for nonlinear state estimation in an actively controlled vehicle suspension application. Despite several significant nonlinear effects in stiffness and damping charac-

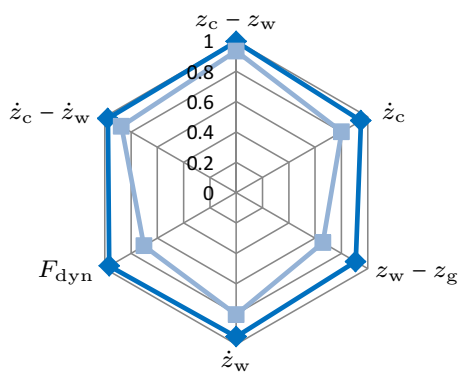

Fig. 4. Estimation performance in simulation and experiment on rough country road profile at $v_{\mathrm{veh}}=50 \mathrm{~km} / \mathrm{h}$ 

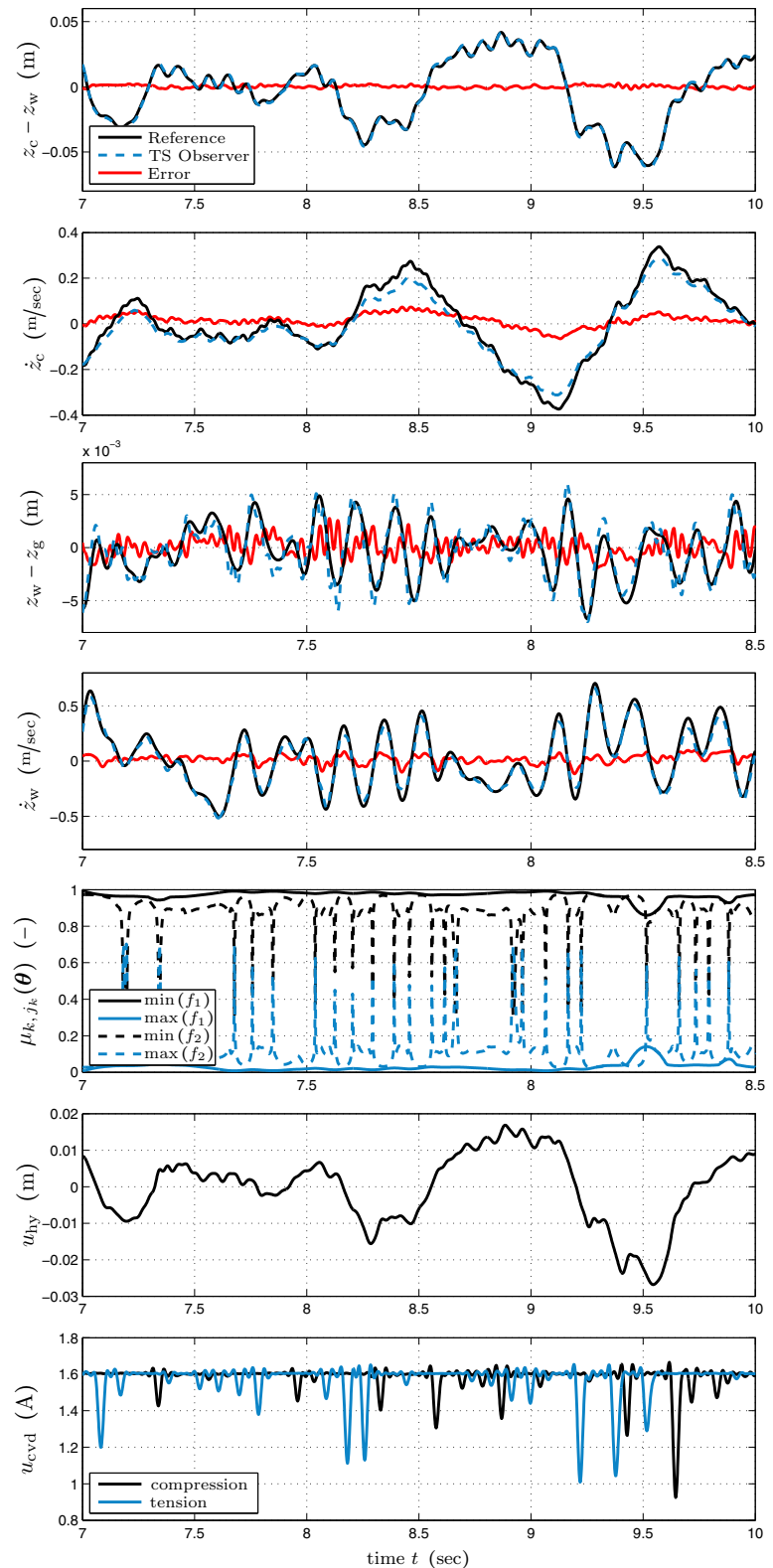

Fig. 5. Measurement results: actively controlled suspension excited by a country road profile $\left(v_{\mathrm{veh}}=50 \mathrm{~km} / \mathrm{h}\right)$

teristics, a number of four linear subsystems is adequate to accurately represent the nonlinear state space model of the hybrid suspension configuration. The observer gains of the linear submodels have been derived based on Kalman filter theory to account for the stochastic nature of the road unevenness and measurement noise. The performance evaluation of the global state observer has been conducted by means of simulations and experiments in a quarter-car framework. Although conventional linear Kalman filtering is used, and thus computational complexity is kept small in comparison to concepts such as EKF and UKF, the TS Kalman filter is able to achieve a high estimation accuracy of $70-99 \%$ for the nonlinear system at hand.

\section{ACKNOWLEDGEMENTS}

The authors thank K. Albert, K.J. Diepold, T. Kloiber, H. Schulte, and S. Spirk for the introduction to the field of TS systems as well as for fruitful discussions and comments.

\section{REFERENCES}

Bergsten, P. (2001). Observers and Controllers for TakagiSugeno Fuzzy Systems. Ph.D. thesis, Örebro University. Deb, K., Pratap, A., Agarwal, S., and Meyarivan, T. (2000). A fast and elitist multiobjective genetic algorithm: NSGA-II. IEEE Trans. Evol. Comput., 6(2), 182 $-197$.

Fleps-Dezasse, M. and Brembeck, J. (2013). Model based vertical dynamics estimation with Modelica and FMI. In Proc. Tth IFAC Symp. Advances in Automotive Control, $331-336$.

Georg, S., Schulte, H., and Aschemann, H. (2012). Control-oriented modelling of wind turbines using a Takagi-Sugeno model structure. In Proc. IEEE WCCI.

Hrovat, D. (1997). Survey of advanced suspension developments and related optimal control applications. Automatica, 33(10), 1781 - 1817.

Kalman, R.E. and Bucy, R.S. (1961). New results in linear filtering and prediction theory. Trans ASME, J Basic Eng, 83, 95 - 108.

Karnopp, D., Crosby, M.J., and Harwood, R.A. (1974). Vibration control using semi-active force generators. Trans ASME, J Eng Ind, 96(2), $619-626$.

Koch, G., Kloiber, T., and Lohmann, B. (2010). Nonlinear and filter based estimation for vehicle suspension control. In Proc. 49th IEEE CDC, $5592-5597$.

Koch, G., Spirk, S., Pellegrini, E., Pletschen, N., and Lohmann, B. (2011). Experimental validation of a new adaptive control approach for a hybrid suspension system. In Proc. 2011 American Control Conference.

Lindgärde, O. (2002). Kalman filtering in semi-active suspension control. In Proc. 15th IFAC World Congress.

Matschinsky, W. (1998). Road Vehicle Suspensions. John Wiley \& Sons, Inc.

Maybeck, P.S. (1979). Stochastic models, estimation, and control, volume 1. Academic Press, Inc. New York.

Mitschke, M. and Wallentowitz, H. (2004). Dynamik der Kraftfahrzeuge. Springer-Verlag Berlin, 4th edition.

Pletschen, N., Spirk, S., and Lohmann, B. (2013). Frequency-selective adaptive control of a hybrid suspension system. In Proc. 7th IFAC Symp. Advances in Automotive Control, 237 - 242.

Sharma, K., Crolla, D.A., and Wilson, D.A. (1994). The design of a fully active suspension system incorporating a Kalman filter for state estimation. In Proc. Int. Conf. on Control, $344-349$.

Simon, D. (2003). Kalman filtering for fuzzy discrete time dynamic systems. Appl Soft Comput, 3(3), $191-207$.

Simon, D. (2006). Optimal State Estimation: Kalman, $H_{\infty}$, and Nonlinear Approaches. John Wiley \& Sons, Inc.

Takagi, T. and Sugeno, M. (1985). Fuzzy identification of systems and its applications to modeling and control. IEEE Trans. Syst., Man, Cybern., 15(1), 116 - 132.

Tanaka, K. and Wang, H.O. (2001). Fuzzy Control Systems Design and Analysis: A Linear Matrix Inequality Approach. John Wiley \& Sons, Inc.

Venhovens, P.J.T. (1994). Optimal Control of Vehicle Suspensions. Ph.D. thesis, Delft University of Technology.

$\mathrm{Yu}, \mathrm{F}$. and Crolla, D.A. (1998). An optimal self-tuning controller for an active suspension. Vehicle Syst Dyn, $29(1), 51-65$.

Zarchan, P. and Musoff, H. (2005). Fundamentals of Kalman Filtering: A Practical Approach. AIAA, Inc. 